\title{
Convergence of the Diagonal Operator-valued Padé Approximants to the Dyson Expansion
}

\author{
F. J. Narcowich and G. D. Allen \\ Department of Mathematics, Texas A \& M University, College Station, Texas 77843, USA
}

\begin{abstract}
The diagonal operator-valued Padé approximants formed from the Dyson expansion to the Schrödinger time-evolution operator are shown to converge everywhere in the complex plane, except on a certain subset of the real axis.
\end{abstract}

The purpose of this paper is to outline a proof of the convergence of the diagonal operator-valued Padé approximants (O.P.A.) formed from the Dyson expansion for the non-relativistic time-evolution operator. This will be carried out by showing that the Dyson expansion belongs to a broad class of operatorvalued analytic functions for which the diagonal O.P.A.'s converge.

Let $U(t, z)$ be the time-evolution operator which solves the Schrödinger equation,

$$
\begin{aligned}
i \frac{d}{d t} U(t, z) & =\left[H_{0}-z V(t)\right] U(t, z) \\
U(0, z) & =I,
\end{aligned}
$$

where $V(t)$ is a bounded, positive-definite operator for all time $t$ and $H_{0}$ is an unperturbed Hamiltonian. It is known [1] that $U(t, z)$ is an entire function of the coupling constant $z$ and that the Dyson expansion [2],

$$
U(t, z)=\sum_{l=0}^{\infty} U_{l}(t) z^{l},
$$

converges for all complex $z$. There are two difficulties concerning this expansion. First of all, for $z$ real, the truncated power series is unitary only to some finite order; secondly, the series may be slowly convergent. On the other hand, Padé approximation techniques can be used to resolve both of these difficulties. In general, the Padé approximants to a series, when they do converge, converge more rapidly than the partial sums; and, in the case of the diagonal $([N / N])$ approximants, they preserve the unitary character of the operator (cf. ZinnJustin, Ref. [3]).

Operator-valued Padé approximants are defined analogously to scalar Padé approximants: Let $A(z)$ be a bounded operator-valued function analytic in a neighborhood of the origin and having the power series expansion

$$
A(z)=\sum_{l=0}^{\infty} A_{l} z^{l} .
$$


The $[N / N]$ O.P.A. to $A(z)$ is defined as the unique rational function $A_{N}(z)=$ $P_{N}(z)\left(Q_{N}(z)\right)^{-1}$ such that $A(z)-A_{N}(z)=D_{2 N+1} z^{2 N+1}+\ldots$, where $P_{N}(z)$ and $Q_{N}(z)$ are polynomials of degree $N$ or less $[3,4]$. The main difficulty in the operator case is with the non-commutativity of the $A_{l}$.

As Zinn-Justin [3] observes, there are several important "invariance" properties of the diagonal operator-valued Padé approximants. Let $\alpha, \beta, \gamma$, and $\delta$ be scalars, then we have:

(a) $\left\{(\alpha A(z)+\beta)(\gamma A(z)+\delta)^{-1}\right\}_{N}=\left(\alpha A_{N}(z)+\beta\right)\left(\gamma A_{N}(z)+\delta\right)^{-1}$.

(b) $A_{N}(\alpha z /(\beta z+\delta))=\{A(\alpha z /(\beta z+\delta))\}_{N}$.

(c) If $A(z)^{\dagger}=A(\bar{z})$, then $A_{N}(z)^{\dagger}=A_{N}(\bar{z})$.

(d) If $A(z)^{\dagger} A(\bar{z})=I$, then $A_{N}(z)^{\dagger} A_{N}(\bar{z})=I$.

In words, the $[N / N]$ Padé approximants are invariant under homographic transformations of the function. They are also invariant under homographic transformations of the variable, provided the origin is fixed. Finally, the diagonal approximants preserve self-adjointness and unitarity.

In the scalar case, the diagonal Padé approximants are known to converge uniformly for Stieltjes series $[5,6]$. Such series arise from functions of the form

$$
f(z)=\int_{0}^{\infty} \frac{d \mu(t)}{t-z}
$$

where $\mu(t)$ is a positive measure with infinitely many points of increase.

Functions of the form given in (3) can be classified according to their mapping properties. In fact, by a theorem of Nevanlinna (cf. Stone, Ref. [7], p. 573) every function which is analytic in the cut complex plane $C-[a, b]$ and preserves the upper and lower half planes has the form

$$
f(z)=\alpha z+\beta+\int_{a}^{b} \frac{d \mu(t)}{t-z} .
$$

By using (4) coupled with properties (a) and (b), which hold for scalar Padé approximants as well, it is possible to show that the diagonal Pade approximants converge uniformly for any function which is analytic in the upper and lower half planes, and in a neighborhood of the origin, and which maps the upper and lower half planes into themselves. Such functions are called $R$-functions (cf. Kac and Krein, Ref. [8]).

The operator-theoretic generalization of $R$-functions was formulated by Wigner [9] in conjunction with the study of the $R$-Matrix (cf. Lane and Thomas, Ref. [10]; Narcowich, Ref. [11]; Wigner, Ref. [12]). Following Wigner's work, we make the

Definition. Let $T(z)$ be an operator-valued ${ }^{1}$ function, analytic in the upper and lower half planes and in a neighborhood of the origin. If also $T(z)$ satisfies

$$
(1 / 2 i)\left(T(z)-T(z)^{\dagger}\right) \cdot \operatorname{Im} z \geqq 0,
$$

we call $T(z)$ an $R$-operator.

\footnotetext{
1 Unless otherwise stated, in what follows all operators belong to the space of bounded linear operators on some Hilbert space $\mathscr{H}$.
} 
For $R$-operators $T(z)$ which are analytic in the cut complex plane $C-[a, b]$, we have the following representation (cf. Allen and Narcowich, Ref. [13]),

$$
T(z)=A z+B+\int_{a}^{b} \frac{d \mu(t)}{t-z},
$$

where $A$ is a bounded positive semi-definite operator, $B$ is a bounded self-adjoint operator and $d \mu(t)$ is a positive strongly countably additive operator-valued measure. [That is, for any Borel set $\Delta, \int_{\Delta} d \mu(t) \geqq 0$, and if $\Delta_{j}$ are mutually disjoint Borel sets and $\Delta=\bigcup_{j=1}^{\infty} \Delta_{j}$, then

$$
\mu(\Delta) \phi=\lim _{n \rightarrow \infty} \sum_{j=1}^{n} \mu\left(\Delta_{j}\right) \phi
$$

for all $\phi \in \mathscr{H}$.]

Bessis and Zinn-Justin [4] have shown that if the underlying space $\mathscr{H}$ is finite dimensional, it is possible to construct the diagonal Padé approximants to (5). By means of continued fractions, they also prove that these approximants converge to $T(z)$ except along the cut. In the infinite dimensional case, the situation is complicated by the presence of the continuous spectrum in the various operators. However, by using a variational principle (cf. Bessis and Talman, Ref. [14]), it is possible to derive a formula for the $[N / N]$ operator-valued Padé approximant which is similar to Nuttall's compact formula (cf. Baker, Ref. [6]), but which avoids assuming invertibility. The convergence of the $[N / N]$ O.P.A.'s to $T(z)$ then follows along much the same path as used by Allen et al. [5] in the scalar case (further details and a complete proof will appear elsewhere). Finally, for any $R$-operator, it is possible to use (a) and (b) to prove that the $[N / N]$ operatorvalued Padé approximants exist, and that they converge on a set which includes the upper and lower half planes and a neighborhood of the origin. Precisely, we have the following

Theorem 1. If $T(z)$ is an R-operator, analytic on $C-\{(-\infty, \beta] \cup[\alpha, \infty)\}$, $\beta<0<\alpha$, then the $[N / N]$ O.P.A.'s converge uniformly in the uniform topology to $T(z)$ on compact sets bounded away from the cut.

From the definition of $R$-operator, it is clear that every such operator is selfadjoint on any portion of the real axis where it is analytic. Thus the modified Cayley transform (cf. Riesz and Sz-Nagy, Ref. [16]) of an $R$-operator

$$
\left(U(z)=-(T(z)-i)(T(z)+i)^{-1},\right.
$$

is unitary on the real axis, away from the cuts. If we assume that $-i$ is never in the spectrum of $T(z)$, then for any $\phi \in \mathscr{H}$,

$$
\left\langle\phi,\left(U^{*} U-1\right) \phi\right\rangle=-4\langle\psi,(\operatorname{Im} T) \psi\rangle,
$$

where $\psi=(T+i)^{-1} \phi$. Hence, $U$ satisfies the following properties:

$$
\begin{aligned}
& U^{*} U \leqq 1 \quad \text { if } \operatorname{Im} z<0, \\
& U^{*} U=1 \quad \text { if } \quad \operatorname{Im} z=0, \quad z \text { not on the cuts } \\
& U^{*} U \geqq 1 \quad \text { if } \quad \operatorname{Im} z>0 .
\end{aligned}
$$


This motivates the following

Definition. Let $U(z)$ be an operator-valued function analytic in the upper and lower half planes and in a neighborhood of the origin. If in addition $U(z)$ satisfies (7.1), (7.2), and (7.3), we call $U(z)$ a $U$-operator.

Given a $U$-operator, if -1 is not in the spectrum of $U(z)$ for $z$ off the real axis and in a neighborhood of the origin, then it is clear that the inverse Cayley transform,

$$
T(z)=i(1-U(z))(1+U(z))^{-1},
$$

is an $R$-operator. By applying Theorem 1 and property (a) of the O.P.A.'s we have

Theorem 2. Given a $U$-operator $U(z)$ such that on the set $S=C-\{(-\infty, \beta] \cup$ $[\alpha, \infty)\}, \beta<0<\alpha, U(z)$ is analytic and -1 does not belong to the spectrum of $U(z)$, then the $[N / N]$ O.P.A.'s converge in the uniform topology to $U(z)$ on $S$.

We are now in a position to prove

Theorem 3. Let $U(t, z)$ be defined by (1), and let $V(t)$ be a bounded measurable operator-valued function of $t$ on $[0, \infty)$ satisfying

$$
V(t) \geqq c>0,
$$

where $c$ is some constant, for all $t \geqq 0$. Then, for any $t \geqq 0$, the $[N / N]$ O.P.A.'s formed from (2) converge to $U(t, z)$ in the uniform topology at each point of the set $S=C-\{(-\infty, \beta] \cup[\alpha, \infty)\}, \quad \beta<0<\alpha$, where the interval $(\beta, \alpha)$ is the smallest interval including the origin for which -1 is not in the spectrum of $U^{\dagger}(t, 0) U(t, z)$.

Proof. Let $\phi$ be any vector in the domain of $H_{0}$. It is well-known that $U(t, z) \phi$ is a strongly differentiable function of $t$ (cf. Kato, Ref. [16]), and that $\|U(t, z) \phi\|^{2}$ is also differentiable in $t$. A short computation shows that

$$
\frac{d}{d t}\|U(t, z) \phi\|^{2}=(-2 \operatorname{Im} z)\langle U(t, z) \phi, V(t) U(t, z) \phi\rangle,
$$

where $\langle$,$\rangle is the inner product in \mathscr{H}$. Using the fact that $V(t) \geqq c$ for all $t$, we have

$$
\frac{d}{d t}\|U \phi\|^{2} \geqq(-2 \operatorname{Im} z) c\|U \phi\|^{2}
$$

if $\operatorname{Im} z<0$ and

$$
\frac{d}{d t}\|U \phi\|^{2} \leqq(-2 \operatorname{Im} z) \mathcal{c}\|U \phi\|^{2}
$$

if $\operatorname{Im} z>0$. From (8) and (9) it immediately follows that

$$
\|U \phi\|^{2} \geqq e^{-2(\operatorname{Im} z) c t}\|\phi\|^{2}, \operatorname{Im} z<0
$$

and

$$
\|U \phi\|^{2} \leqq e^{-2(\operatorname{Im} z) c t}\|\phi\|^{2}, \operatorname{Im} z>0 .
$$

By the continuity of $U,(10)$ and (11) hold for all vectors in $\mathscr{H}$. Hence, $U(t, z)$ is a $U$-operator. Since multiplication by $U(t, 0)^{\dagger}$ does not affect $(10)$ and $(11)$, the 
operator $\tilde{U}(t, z)=U^{\dagger}(t, 0) U(t, z)$ is also a $U$-operator. Moreover, for $t \geqq 0,(10)$ and (11) imply that -1 is not in the spectrum of $\tilde{U}$ off the real axis. Also, since $\tilde{U}(t, 0)=1,-1$ cannot be in the spectrum of $\tilde{U}(t, z)$ in a neighborhood of $z=0$. By Theorem 2, the diagonal O.P.A.'s converge to $\tilde{U}(t, z)$ in $S$. Finally, the diagonal O.P.A.'s to $U(t, z)$ converge to $U(t, z)$ on $S$ because they are simply the $[N / N]$ O.P.A.'s to $\tilde{U}(t, z)$ multiplied by $U(t, 0)$. This completes the proof.

We remark that the condition $V(t) \geqq c$ can be very simply achieved by the addition of a constant to the perturbing potential. This merely produces an oscillatory term in the time-evolution operator.

It is not known if the O.P.A.'s converge at points $z$ for which $U^{\dagger}(t, 0) U(t, z)$ has -1 in its spectrum. However, by way of analogy, it can be shown (Narcowich, Ref. [17]) that if, for $e^{i z}$, we consider the function

$$
g(z)=i\left(1-e^{i z}\right)\left(1+e^{i z}\right)^{-1},
$$

then the complex-valued diagonal Padé approximants to $e^{i z}$ do converge for all $z$, even at the poles of $g(z)$. Thus it is probable that the diagonal O.P.A.'s converge to $U(t, z)$ for all $z$.

\section{References}

1. Hepp, K.: Theorie de la renormalization. Lecture notes in physics, Vol. 2, p. 30, Berlin-HeidelbergNew York: Springer 1969

2. Gottfried, K.: Quantum mechanics. New York: Benjamin 1966

3. Zinn-Justin, J.: Phys. Lett. C1, 55 (1971)

4. Basdevant, J.L., Bessis, D., Zinn-Junstin, J.: Nuovo Cimento 60A, 185 (1969); Mathematical Appendices

5. Allen, G.D., Chui, C.K., Madych,W.R., Narcowich,F.J., Smith,P.W.: Bull. Australian Math. Soc. 10, 263 (1974)

6. Baker, G. A., Jr.: Adv. Theor. Phys. 1, 1 (1965)

7. Stone, M. H.: Linear transformations in Hilbert space. Providence: Amer. Math. Soc. 1932

8. Kac, I. S., Krein, M. G. : Amer. Math. Soc. Transl. (2), 103, 1 (1974)

9. Wigner, E. P.: Causality, $R$-matrix, and collision matrix. In: Rendiconti della Scuola Internazionale di Fisica «E. Fermi» XXIX Corso

10. Lane, A. M., Thomas, R. G.: Rev. Mod. Phys. 30, 357 (1958)

11. Narcowich,F.J.: Mathematical theory of the $R$-matrix. Parts I and II. J. Math. Phys. 15, 1626, 1635 (1974)

12. Wigner, E.P.: Phys. Rev. 70, 15 (1946)

13. Allen, G.D., Narcowich,F.J.: On the representation and approximation of a class of operatorvalued analytic functions. Bulletin Am. Math. Soc. 81, 410 (1975)

14. Bessis, D., Talman, J. D.: Rocky Mountain J. Math. 4, 151 (1974)

15. Riesz,T., Sz-Nagy, B.: Functional analysis. New York: Ungar 1965

16. Kato, T.: Perturbation theory for linear operators. Berlin-Heidelberg-New York: Springer 1966

17. Narcowich, F. J.: Unpublished

Communicated by A. S. Wightman

Received November 5, 1974; in revised form May 26, 1975 
